\title{
Comparison Study of Compliance with Medication and Foot Care in Type 2 Diabetic Patients
}

\section{Vivek Gaurang Vyas*}

Department of Pharmacy Practice, SJM College of Pharmacy, Ahmedabad, Gujarat, India

\begin{abstract}
Introduction: The term "diabetes mellitus" describes a metabolic disorder of multiple etiology characterized by chronic hyperglycemia with disturbances of carbohydrate, fat and protein metabolism resulting from defects in insulin secretion, insulin action, or both. Adherence has been defined as the active, voluntary, and collaborative involvement of the patient in a mutually acceptable course of behavior to produce a therapeutic result. This definition implies that the patient has a choice and that both patients and providers mutually establish treatment goals and the medical regimen.
\end{abstract}

Objectives: To assess the compliance for medication and foot care.

Methodology: An interventional study was carried out in people having diabetes for atleast 2 years who visited $\mathrm{BMCH}$, Chitradurga, Karnataka, for a period of six month. A total of 251 patients were enrolled in the study. SPSS software was used to calculate the statistical estimation. Student t-test was used to analyze different variables.

Results: Adherence to medication revealed that 70 (27.89\%) patients showed low adherence, $179(71.34 \%)$ moderate adherence and $2(0.79 \%)$ showed high adherence at the baseline. Follow up revealed $3(1.19 \%)$ patients were found to be low adherent, 247 (99.40\%) moderate adherence and 1 patient $(0.39 \%)$ high adherent.

Compliance to foot care showed that 242 (96.41\%) poor compliance, $7(2.78 \%)$ moderate compliance and 2 $(0.796 \%)$ high compliance at baseline. At follow-up it revealed that $144(57.37 \%)$ showed poor compliance, 104 $(41.43 \%)$ moderate compliance and $3(1.195 \%)$ high compliance.

Conclusion: As diabetes is a lifelong disease and taking medicines daily is difficult for patients. Pharmacist educating patient about importance of medication adherence and foot care to prevent further worsening the disease helps the patient to control blood glucose level and stay healthy.

Keywords: Diabetes mellitus; Adherence; Interventional study

\section{Introduction}

Diabetes is a metabolic syndrome characterized by inappropriate high blood glucose result in the form of either low level of insulin or in the form of abnormal resistance to insulin effect coupled with inadequate level of insulin secretion to compensate. The characteristic symptoms are: Excessive urine production (polyuria), Increased hunger (polyphagia), Increased thirst (polydipsia) and Blurred vision [1]

International Diabetes Federation (IDF) 2013 projects the prevalence of diabetes in India.

India is one of the 6 countries of the IDF SEA region.

$>387$ million people have diabetes in the world and 75 million people in the SEA Region; by 2035 this will rise to 123 million.

$>$ There were 66.8 million cases of diabetes in India in 2014 [2].

The definition of adherence according to WHO is the extent to which a person's behavior - taking medication, following a diet and/or performing lifestyle changes- corresponds with agreed recommendations from the health care provider.

In every aspect the primary goal in the management of chronic disease is the improvement of the patients' HR-QoL [3].

There are seven essential self-care behaviors in people with diabetes which predict good outcomes namely healthy eating, being physically active, monitoring of blood sugar, compliant with medications, good problem-solving skills, healthy coping skills and risk-reduction behaviors. All these seven behaviors have been found to be positively correlated with good glycemic control, reduction of complications and improvement in quality of life [4].

Medication adherence usually refers to whether patients take their medications as prescribed (e.g., twice daily), as well as whether they continue to take a prescribed medication. Medication adherence behavior has thus been divided into 2 main concepts, namely, adherence and persistence. Although conceptually similar, adherence refers to the intensity of drug use during the duration of therapy, whereas persistence refers to the overall duration of drug therapy. Medication adherence is a growing concern to clinicians, healthcare systems, and other stakeholders (e.g. payers) because of mounting evidence that non-adherence is prevalent and associated with adverse outcomes and higher costs of care [5].

Diabetic foot is one of the most significant and devastating

*Corresponding author: Vivek Gaurang Vyas, Doctor of Pharmacy, Department of Pharmacy Practice, SJM College of Pharmacy, 20 zanzar flat, judges bunglow main road, near SG highway, Ahmedabad-360018, Gujarat, India, Tel: 9986082997; E-mail: drvivekvyas@live.in

Received July 29, 2015; Accepted August 19, 2015; Published August 24, 2015

Citation: Vyas VG (2015) Comparison Study of Compliance with Medication and Foot Care in Type 2 Diabetic Patients. J Diabetes Metab 6: 593. doi:10.4172/21556156.1000593

Copyright: (C) 2015 Vyas VG. This is an open-access article distributed under the terms of the Creative Commons Attribution License, which permits unrestricted use, distribution, and reproduction in any medium, provided the original author and source are credited. 
complications of diabetes, and is defined as a foot affected by ulceration that is associated with neuropathy and/or peripheral arterial disease of the lower limb in a patient with diabetes.

The prevalence of diabetic foot ulceration in the diabetic population is $4-10 \%$; the condition is more frequent in older patients. It is estimated that about $5 \%$ of all patients with diabetes present with a history of foot ulceration, while the lifetime risk of diabetic patients developing this complication is $15 \%$.

Neuropathic wounds are more likely to heal over a period of 20 weeks, while neuroischemic ulcers take longer and will more often lead to limb amputation. It has been found that $40-70 \%$ of all nontraumatic amputations of the lower limbs occur in patients with diabetes [6].

The main goals of diabetic foot care involve a combination of preventive strategies, including patient education, involvement, and adherence to physician recommendations, as well as maintaining tight glycemic control and performing routine skin, foot, and nail inspections.

Left untreated, bacterial and fungal infections, as well as foot ulcers, can lead to amputation.

The American Diabetes Association's Standards of Medical Care, released in 2014, include the following foot care recommendations:

- Patients with diabetes should obtain an annual comprehensive foot examination to identify risk factors predictive of ulcers and amputations.

- Patients with diabetes should receive general foot self-care education from their primary health care provider.

- A multidisciplinary approach is recommended for patients with foot ulcers and those at high risk for foot complications, especially individuals with a history of prior ulcer or amputation.

- Patients, who smoke and/or have structural abnormalities, as well as those with a history of lower extremity complications, should be referred to foot care specialists for ongoing preventive care and lifelong surveillance [7].

\section{Objective}

This study is aimed to assess adherence to medication and identify the factors that are associated with non-adherence in type-2 DM patients, because non-adherence could lead to worsening of disease and increased risk of complications. Pharmacist's intervention may have a greater impact on the health of the population than any improvement in specific medical treatments. The aim of this large population-based study is to evaluate the self-reported medication adherence and foot care in patients with type-2 DM.

\section{Methodology}

A prospective interventional study conducted over a period of six months using questionnaires as a tool, was conducted in Basaveshwara Medical College Hospital \& Research Centre, Chitradurga. Patient with diabetes for at least 2 years, with diabetic complications and other comorbid conditions were included in study. Data was collected through personal interview with patients. All the patients who admitted to the General Medicine department were reviewed daily to identify the patients diagnosed with Diabetes or with other co morbidities. The patients who met the study criteria were enrolled in the study.
Patients are enrolled in to the project by providing questionnaires and data is collected at the initial visit, further patient counseling is given using PILs and foot care pictograms. Follow up all the enrolled patients is done after 3months with same questionnaires; compliance is analyzed by evaluating the baseline and follow-up data. Consents are obtained before the study. Ethical clearance from Institutional Ethical Committee, of Basaveshwara Medical College Hospital \& Research Centre, Chitradurga was obtained prior to the study.

\section{Results}

The filled data collection forms were analyzed for medication adherence and self-foot care after counselling provided. A total of 327 patients with a history of DM for at least 2 years were enrolled in the study. The newly detected diabetic patients were not enrolled in the study.

\section{Compliance to medication}

Adherence to medication was assessed using MMAS-8 questionnaire. After analyzing the questionnaire it was found to be that out of 251 subjects 70 (27.89\%) patients showed low adherence, 179 (71.34\%) moderate adherence and 2 (0.79\%) showed high adherence at the baseline with a mean of $2.10(\mathrm{SD} \pm 1.461)$ after using paired sample statistics.

Follow up was conducted after a period of 3 months which revealed $3(1.19 \%)$ patients were found to be low adherent, $247(99.40 \%)$ moderate adherence and 1 patient $(0.39 \%)$ high adherence with a mean of $1.20(\mathrm{SD} \pm 0.639)$.

By analyzing MMAS scale of base line and follow up using a 'paired $t$ test' resulted in a mean of $0.904(S D \pm 1.102)$ and DF 250. The calculated $t$ value was higher than the table value of $\mathrm{DF}$, which suggests significance and Null Hypothesis fails (Figure 1).

\section{Compliance to foot-care}

Out of 251 patients 242 (96.41\%) showed poor compliance, $7(2.78 \%)$ moderate compliance and $2(0.796 \%)$ high compliance at baseline. At follow-up it revealed that 144 (57.37\%) showed poor compliance, 104 (41.43\%) moderate compliance and 3 (1.195\%) high compliance. By analyzing compliance to foot-care at baseline and follow using paired t-test revealed foot-care (mean \pm SD) as $(1.77 \pm$ 1.54) and DF 250. The calculated $t$-value was higher table value of DF, which shows significance and null hypothesis is rejected (Figure 2).

\section{Medication adherence}

\section{NUMBER OF PATIENTS DURING BASE LINE}

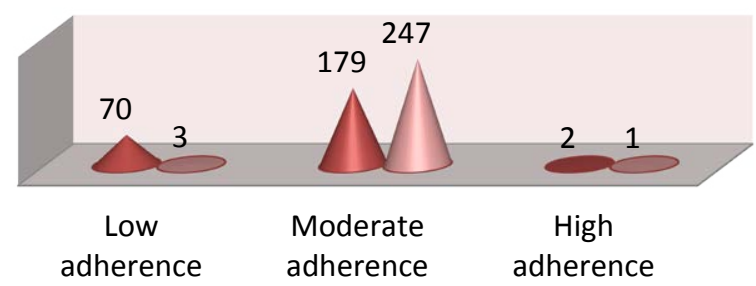

Figure 1: Distribution of patients according to medication adherence. 


\section{Compliance to Foot care}

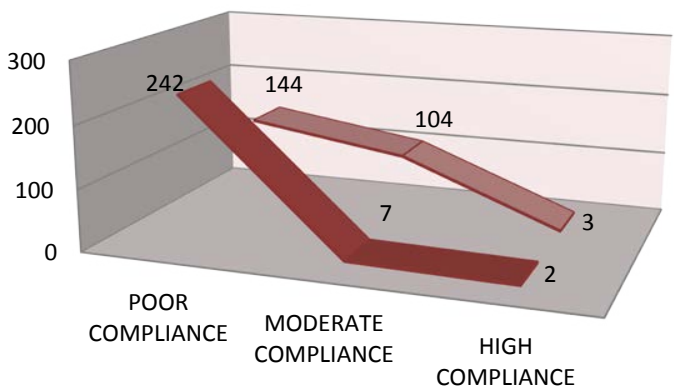

Figure 2: Distribution of diabetic patients according to foot care compliance.

\section{Discussion}

Adherence has been defined as the active, voluntary, and collaborative involvement of the patient in a mutually acceptable course of behavior to produce a therapeutic result. This definition implies that the patient has a choice and that both patients and providers mutually establish treatment goals and the medical regimen. A merged version of the definitions of Haynes and Rand [8,9] "the extent to which a person's behaviour - taking medication, following a diet, and/or executing lifestyle changes, corresponds with agreed recommendations from a health care provider." The adherence project has adopted the following definition of chronic diseases "Diseases which have one or more of the following characteristics: they are permanent, leave residual disability, are caused by nonreversible pathological alteration, require special training of the patient for rehabilitation, or may be expected to require a long period of supervision, observation or care" [10].

In this study, we have examined adherence to medication and compliance to foot-care behavior at a tertiary care setting to type-2 diabetes and tried to explore whether pharmacist intervention had any role in improving in the compliance rate.

The findings are valuable, as no such study has ever been reported from this area. It documents the efficacy of guidance on foot-care behaviour to all the people with diabetes who visited the hospital.

This study provided valuable evidence that the advice given on medication adherence, and advice on foot care is effective in modifying self-care behaviour and thus has a great potential on the outcomes of the treatment.

Similar study conducted on South Asian people living in developed countries has shown that they have relatively poorer compliance rate [11-13]. Our study reported moderate compliance at the baseline but on pharmacist intervention, the compliance rate was higher.

Ayman et al., studied on impact of education programmed on patient-anxiety, depression, glycemic control and adherence to selfcare and medication in type-2 diabetes mellitus [14]. The study resulted in significant improvement in patients' dietary plan, physical exercise, self-monitoring of blood glucose (SMBG), $\mathrm{HbA}_{1} \mathrm{C}$, adherence to medication. This study has also followed the procedure of providing education to patients in local language including pamphlets and handouts. One on one counseling was arranged which resulted in significant improvement in patients medication taking behaviour and foot care behaviour.

Mumu SJ et al, Non-adherence to life-style modification and its factors among type 2 diabetic patients. They studied on proportion of non-adherence to life-style modification and factors associated. Non-adherence rate were assessed for: foot care (70\%) [15]. This study reported the noncompliance to foot care (96.41\%).

Farah $\mathrm{M}$ et al., Conducted a comparative study on Cognitive status and foot self-care practice in overweight diabetics, engaged in different levels of physical activity [16]. This study though did not compare foot self-care practice with that of cognitive status; it reported that the reason for poor compliance to foot care was lack of patient education and awareness.

\section{Conclusions}

As diabetes is a lifelong disease and taking medicines daily is difficult for patients. Pharmacist educating patient about importance of medication adherence and foot care to prevent further worsening the disease helps the patient to control blood glucose level and stay healthy.

\section{References}

1. Ho PM, Bryson CL, Rumsfeld JS (2009) Medication adherence: its importance in cardiovascular outcomes. Circulation 119: 3028-3035.

2. www.idf.org. accessed on 25-7-2015.

3. Saleh F, Mumu SJ, Ara F, Hafez MA, Ali L (2014) Non-adherence to self-care practices \& medication and health related quality of life among patients with type 2 diabetes: a cross-sectional study. BMC Public Health 14: 431.

4. Shrivastava SR, Shrivastava PS, Ramasamy J (2013) Role of self-care in management of diabetes mellitus. J Diabetes Metab Disord 12: 14.

5. Ho PM, Bryson CL, Rumsfeld JS (2009) Medication adherence: its importance in cardiovascular outcomes. Circulation 119: 3028-3035.

6. Alexiadou K, Doupis J (2012) Management of diabetic foot ulcers. Diabetes Ther 3: 4.

7. Yvette CT (2014) Diabetic Foot Care: The Importance of Adherence.

8. Haynes RB (1979) Determinants of compliance: The disease and the mechanics of treatment. Johns Hopkins University Press, Baltimore MD.

9. Rand CS (1993) Measuring adherence with therapy for chronic diseases: implications for the treatment of heterozygous familial hypercholesterolemia. Am J Cardiol 72: 68D-74D.

10. Dictionary of health services management ( $2^{\text {nd }}$ edtn) Owing Mills, MD, National Health Publishing, 1982.

11. Banerjee AT, Gupta M, Singh N (2007) Patient characteristics, compliance, and exercise outcomes of South Asians enrolled in cardiac rehabilitation. $J$. Cardiopulm. Rehabil Prev 27: 212-218.

12. Butterworth JR, Banfield LM, Iqbal TH, Cooper BT (2004) Factors relating to compliance with a gluten-free diet in patients with coeliac disease: comparison of white Caucasian and South Asian patients. Clin Nutr 23: 1127-1134.

13. Pardhan S, Mahomed I (2004) Knowledge, self-help and socioeconomic factors in South Asian and Caucasian diabetic patients. Eye (Lond) 18: 509-513.

14. Al Hayek AA, Robert AA, Al Dawish MA, Zamzami MM, Sam AE, et al. (2013) Impact of an education program on patient anxiety, depression, glycemic control, and adherence to self-care and medication in Type 2 diabetes. J Family Community Med 20: 77-82

15. Mumu SJ, Saleh F, Ara F, Afnan F, Ali L (2014) Non-adherence to life-style modification and its factors among type 2 diabetic patients. Indian $\mathrm{J}$ Public Health 58: 40-44.

16. Farah M, Mohsen $H$, Mohsen KN (2014) Cognitive status and foot self-care practice in overweight diabetics, engaged in different levels of physical activity. Journal of Diabetes and Metabolic Disorders 13: 31. 